\title{
Controlling Chaos in DC/DC Converters Using Optimal Resonant Parametric Perturbation
}

\author{
Yufei Zhou ${ }^{1}$, Herbert H. C. Iu ${ }^{2}$, Chi K. Tse ${ }^{3}$ and Jun-Ning Chen ${ }^{1}$ \\ ${ }^{1}$ Department of Electronics, Anhui University, Hefei, Anhui, China \\ ${ }^{2}$ School of Electrical, Electronic and Computer Engineering, The University of Western Australia, Australia \\ ${ }^{3}$ Department of Electronic and Information Engineering, Hong Kong Polytechnic University, Hong Kong, China
}

\begin{abstract}
The method of resonant parametric perturbation is a simple non-feedback chaos control method which is easy to implement in practice. In this paper, an optimal strategy is applied to stabilize an unstable orbit in a chaotically operating current-mode controlled buck-boost converter. Optimal values of initial phase angles are computed corresponding to minimum perturbation amplitudes. With this optimal perturbation, the converter operating in a chaotic regime can be controlled to operate in an unstable period-1 orbit that exists in the original chaotic attractor.
\end{abstract}

\section{INTRODUCTION}

Power converters are practical nonlinear systems which find applications in many electronic products and equipments. It has been shown that the operation of power converters can easily become chaotic when they fail to maintain their normal periodic operation [1]. Thus, the ability to avoid chaos is almost a basic feature of all existing practical control strategies, although practicing engineers may not always be aware of such a perspective. Recently many methods have been proposed for controlling chaos in nonlinear systems. They can be classified into two general categories [2], namely, feedback control methods and non-feedback control methods. Comparing to the feedback type of control, the non-feedback type of control is easier to implement, but it does not always lead to the stabilization of an unstable period-1 orbit that exists in the original chaotic attractor.

In this paper, we consider a resonant parametric perturbation method for controlling chaos in a current-mode controlled buck-boost converter. With an optimal strategy, we achieve the same control results that can be obtained from the feedback type of control. Specifically we make a chaotic buck-boost converter operate in an unstable period-1 orbit that exists in the original chaotic attractor. In Section II, we will introduce the circuit operation of the buck-boost converter under current-mode control and some typical bifurcation routes. In Section III, an optimal resonant parametric perturbation method will be introduced, and in this application example, we show that an unstable period-1 orbit in the

This work was supported by the National Natural Science Foundation of China(60402001) and in part by the Hong Kong RGC under Grant PolyU 5241/03E. chaotic attractor can be stabilized, as could be achieved by a more complicated feedback chaos control method.

\section{CURRENT-MODE CONTROLled BuCK-BoOsT CONVERTER}

\section{A. Basic Operation}

A buck-boost converter under current-mode control [3] is shown in Fig. 1. The switch is turned on periodically by the clock, and off according to the output of a comparator that compares the inductor current $i_{\mathrm{L}}$ with a current reference $I_{\text {ref. }}$. Specifically, while the switch is on, the inductor current climbs up, and as it reaches $I_{\text {ref }}$, the switch is turned off, thereby causing the inductor current to ramp down until the next clock comes. Thus, according to the switch state $u$, the circuit will have two topologies that can be described by the following differential equations:

$$
\left\{\begin{array}{cl}
\dot{x}=A_{\text {on }} x+B_{\text {on }} E & \mathrm{G} \text { is on } \\
\dot{x}=A_{\text {off }} x+B_{\text {off }} E & \mathrm{G} \text { is off }
\end{array}\right.
$$

where $x$ denotes the state variables, i.e. $x=\left[i_{\mathrm{L}}, v_{\mathrm{O}}\right]^{T}$, the A's and B's are the system matrices given by

$$
\begin{aligned}
& A_{\text {on }}=\left[\begin{array}{cc}
0 & 0 \\
0 & -1 / R C
\end{array}\right], \quad B_{\text {on }}=\left[\begin{array}{c}
1 / L \\
0
\end{array}\right] \\
& A_{\text {off }}=\left[\begin{array}{cc}
0 & 1 / L \\
-1 / C & -1 / R C
\end{array}\right], \quad B_{\text {off }}=\left[\begin{array}{l}
0 \\
0
\end{array}\right]
\end{aligned}
$$

Using the above equations, "exact" cycle-by-cycle simulation can be performed use SIMULINK model. The parameters are chosen as: $E=10 \mathrm{~V}, L=1 \mathrm{mH}, C=4 \mu \mathrm{F}, R=20 \Omega$, $T=50 \mu \mathrm{s}\left(f_{\mathrm{s}}=20 \mathrm{kHz}\right), I_{\mathrm{ref}}=0.5-4.5 \mathrm{~A}$.

\section{B. Chaotic Behavior}

The afore-described buck-boost converter has been shown previously to exhibit period-doubling bifurcation 
when some parameters are varied [3]. A typical bifurcation diagram is shown in Fig. 2, where $I_{\text {ref }}$ is chosen as the bifurcation parameter, and the variation of the largest Lyapunov exponent is shown in Fig. 2(b). As shown in Fig. 2(a), the buck-boost converter goes through a typical period-doubling bifurcation route, and eventually it enters the chaotic regime when $I_{\text {ref }}$ exceeds about 1.45 A. Fig. 3 shows the phase portrait and Poincare section for the case of $I_{\text {ref }}=4$ A, which corresponds to the chaotic operation.

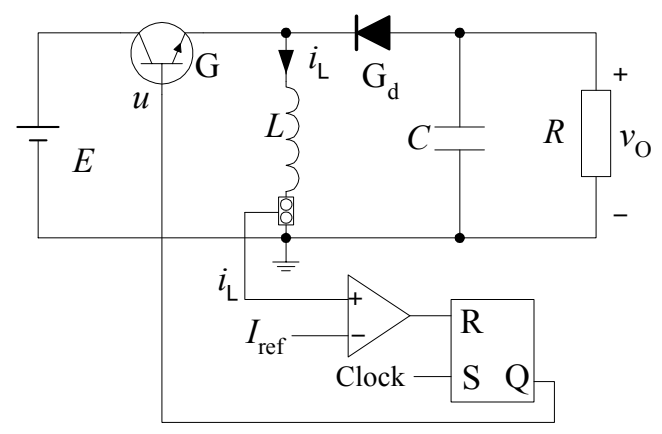

(a)
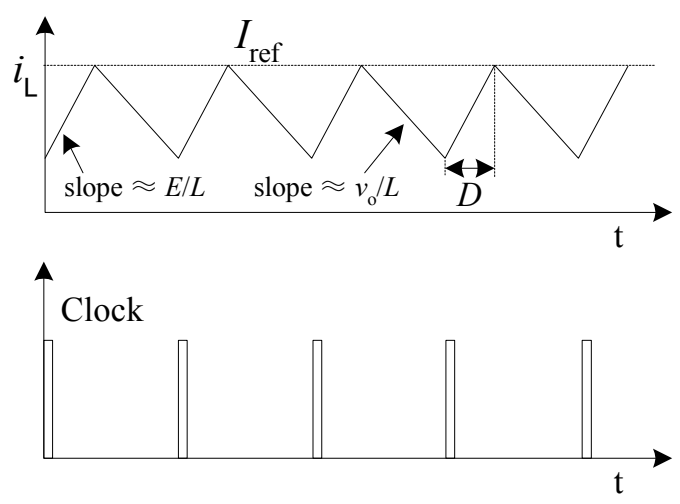

(b)

Fig. 1: Current-mode controlled buck-boost converter. (a) Schematic diagram; (b) operation waveform.

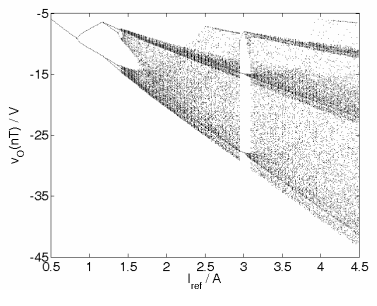

(a)

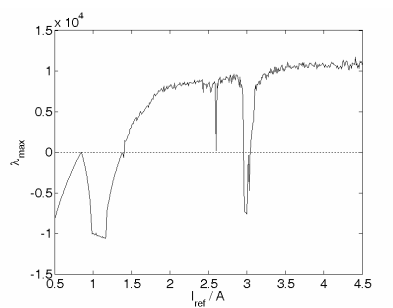

(b)
Fig. 2: Bifurcation of current-mode controlled buck-boost converter. (a) Simulated bifurcation diagram with $I_{\text {ref }}$ as the bifurcation parameter; (b) largest Lyapunov exponent.

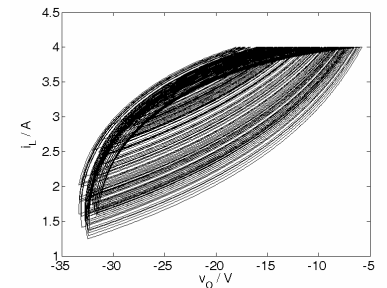

(a)

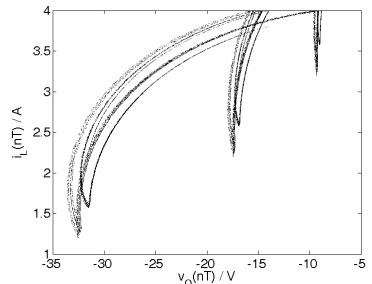

(b)
Fig. 3: Chaotic operation of current-mode controlled buckboost converter. (a) phase portrait; (b) Poincaré section.

\section{Control of Chaos In CuRRent-Mode CONTROLLED BUCK-BOOST CONVERTER BY RESONANT PARAMETERIC PERTURBATION}

\section{A. Review of Resonant Parametric Perturbation}

The usual procedure of resonant parametric perturbation is to choose a parameter that strongly affects the system's dynamics and can be easily varied. Suppose this parameter is $c$, it is then perturbed with the function $[1+A \sin (2 \pi f t)]$, where $A$ « 1 and $f$ is the perturbation frequency to be chosen. Effectively, we are replacing $c$ by $c[1+A \sin (2 \pi f t)]$ such that the largest Lyapunov exponent is reduced to below zero. This approach has been used by Lima and Pettini for stabilizing a chaotic Duffing-Holmes system [4]. In particular, it has been shown that when the perturbation frequency $f$ resonates with the periodic driving frequency, say $f_{\mathrm{s}}$, the largest Lyapunov exponent will approach zero from positive, and eventually chaos subsides and the periodic state emerges as the largest Lyapunov exponent falls further below 0 .

\section{B. Application to Buck-Boost Converter}

From Fig. 2 we can see the first bifurcation occurs at about $I_{\text {ref }}=0.84 \mathrm{~A}$, which corresponds to the situation of losing stability. For current-mode controlled buck-boost converter, the circuit will lose stability when duty cycle $D$ exceeds 0.5. Similar to the treatment in [5], we can get the critical value of $I_{\text {ref }}$ corresponding to stable operation

$$
I_{\mathrm{ref}}<\frac{E}{R}\left[\frac{D R}{2 \xi}+\frac{D}{(D-1)^{2}}\right]_{D=0.5}=\frac{E}{R}\left[\frac{R}{4 \xi}+2\right]=I_{\mathrm{ref}, \mathrm{c}}
$$

where $\xi=L / T$.

As indicated before, when $I_{\text {ref }}=4 \mathrm{~A}$, the buck-boost converter operates in the chaotic regime with the largest Lyapunov exponent being equal to 10733 . We wish to control it to operate on the (now unstable) period-1 orbit. When the method of resonant parametric perturbation is applied, we need to select the perturbing parameter. Apparently the current reference $I_{\text {ref }}$ is a convenient choice as it can be changed easily. Essentially we replace $I_{\text {ref }}$ by the perturbed current reference $\widetilde{I}_{\text {ref }}$, i.e.,

$$
\widetilde{I}_{\text {ref }}=I_{\text {ref }}[1+A \sin (2 \pi f t)]
$$


where $A$ is the perturbation amplitude, $f$ is the perturbation frequency and should be set to switching frequency $f_{\mathrm{s}}$. Thus, the term $A \sin (2 \pi f t)$ is the resonant perturbation applied to $I_{\text {ref. }}$ With this perturbation, the chaotic converter can be controlled when amplitude $A$ exceeds 0.076 .

\section{Optimal Resonant Parametric Perburation}

In this subsection, we will describe an optimal resonant parametric perturbation [6] for controlling chaos in the buckboost converter. This method will use the smallest effective perturbation amplitude $A$, while achieving the same control results as that of the feedback chaos control method. In other words, the converter can be stabilized in the unstable period1 orbit that exists in the chaotic attractor with minimum control effort. Simply, we will replace the perturbed current reference (4) by:

$$
\widetilde{I}_{\text {ref }}=I_{\text {ref }}[1+A \sin (2 \pi f t+\theta)]
$$

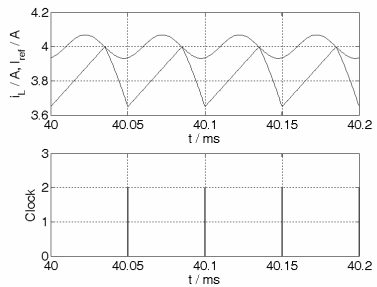

(a)

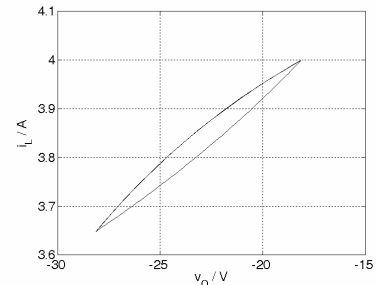

(b)
Fig. 4: Waveforms of the chaotic buck-boost converter controlled by optimal resonant parametric perturbation. (a) timedomain waveforms; (b) phase portrait.

In (5), there are two adjustable parameters, namely amplitude $A$ and initial phase angle $\theta$. Basically we find the optimal $\theta$ for stabilizing the unstable orbit with minimum $A$. Firstly, from simulations, we find the optimal initial phase as 5.04 and the corresponding smallest $A$ as 0.017 ; that is, when initial phase $\theta$ is selected as the optimal value (5.04), the chaotic converter can be controlled to work on the period-1 orbit, as shown in Fig. 4. We can verify by computation that this orbit is exactly the unstable period-1 orbit in the chaotic attractor with the negative largest Lyapunov exponent equal to -3055 . The perturbation amplifier $A$ is 0.017 , which is the smallest value. From the time-domain waveforms shown in Fig. 4(a), we can see that the point where $i_{\mathrm{L}}$ touches $\widetilde{I}_{\text {ref }}$ is exactly the unperturbed current reference value $\left(I_{\text {ref }}=4 \mathrm{~A}\right)$ and at this point the gradient of the reference current is a minimum. Thus, the perturbation has the least influence on the converter, and the same control result as the method of feedback can be achieved. Inspecting the waveforms in Fig. 4(a), we can derive the relation between $D$ and $\theta$ :

$$
\theta=2 \pi-2 \pi(D-0.5)=2 \pi(1.5-D)
$$

Similar to the treatment described in [5], we inspect the inductor current $i_{\mathrm{L}}$ and the perturbed current reference $\widetilde{I}_{\text {ref }}$, and get

$$
\left\{\begin{array}{c}
\frac{\widetilde{I}_{\mathrm{ref}}-i_{\mathrm{n}}}{D T}=\frac{E}{L} \\
\frac{\widetilde{I}_{\mathrm{ref}}-i_{\mathrm{n}+1}}{(1-D) T}=\frac{-v_{o}}{L}
\end{array}\right.
$$

where $D$ is the duty cycle, and the perturbation style of this equation is:

$$
\left\{\begin{array}{c}
\frac{I_{\text {ref }}\{1+A \sin [2 \pi(D+\delta D)+\theta]\}-\left(i_{\mathrm{n}}+\delta i_{\mathrm{n}}\right)}{(D+\delta D) T}=\frac{E}{L} \\
\frac{I_{\text {ref }}\{1+A \sin [2 \pi(D+\delta D)+\theta]\}-\left(i_{\mathrm{n}+1}+\delta i_{\mathrm{n}+1}\right)}{[1-(D+\delta D)] T}=\frac{-v_{o}}{L}
\end{array}\right.
$$

Considering only the transient variables, (8) can be written as

$$
\left\{\begin{array}{l}
2 \pi A I_{\text {ref }} \cos (2 \pi D+\theta) \delta D-\delta i_{\mathrm{n}}=\frac{E \delta D T}{L} \\
2 \pi A I_{\text {ref }} \cos (2 \pi D+\theta) \delta D-\delta i_{\mathrm{n}+1}=\frac{v_{o} \delta D T}{L}
\end{array}\right.
$$

or

$$
\left\{\begin{array}{c}
-m_{c} f \delta D-\delta i_{\mathrm{n}}=\frac{E \delta D T}{L} \\
-m_{c} f \delta D-\delta i_{\mathrm{n}+1}=\frac{v_{o} \delta D T}{L}
\end{array}\right.
$$

where $m_{\mathrm{c}}=-\frac{d \widetilde{I}_{\mathrm{ref}}}{d t}=-2 \pi A f I_{\mathrm{ref}} \cos (2 \pi D+\theta)$.

Combining the above equations, we have the following iterative function:

$$
\begin{aligned}
\delta i_{\mathrm{n}+1} & =\left[\frac{M_{c}}{M_{c}+1}+\frac{v_{o}}{\left(M_{c}+1\right) E}\right] \delta i_{\mathrm{n}} \\
& =\left[\frac{M_{c}}{M_{c}+1}+\frac{D}{\left(M_{c}+1\right)(D-1)}\right] \delta i_{\mathrm{n}}
\end{aligned}
$$

where

$$
M_{c}=L m_{c} / E=-2 \pi A f L I_{\mathrm{ref}} \cos (2 \pi D+\theta) / E
$$

For the optimal chaos control shown in Fig. 4, we have

$$
M_{c}=-2 \pi A f L I_{\text {ref }} \cos (\pi) / E=2 \pi A f L I_{\text {ref }} / E
$$

Now using (11) we can get the eigenvalue or characteristic multiplier $J$ as

$$
J=\frac{M_{c}}{M_{c}+1}+\frac{D}{\left(M_{c}+1\right)(D-1)}
$$




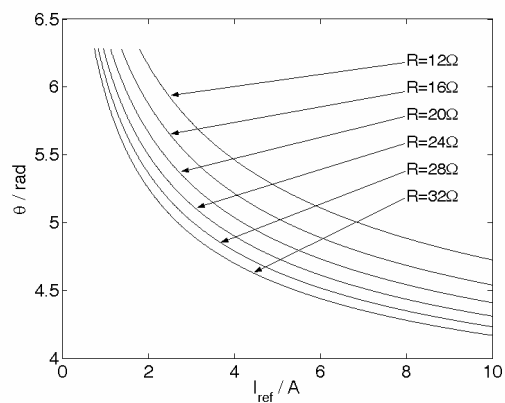

(a)

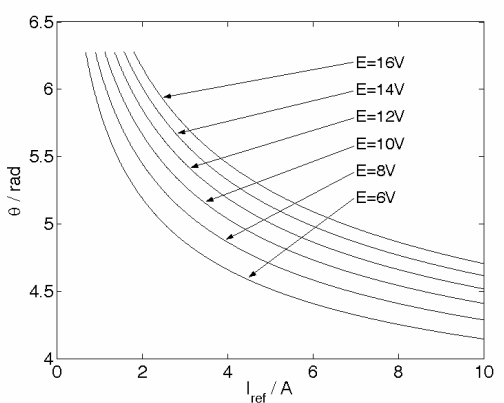

(b)

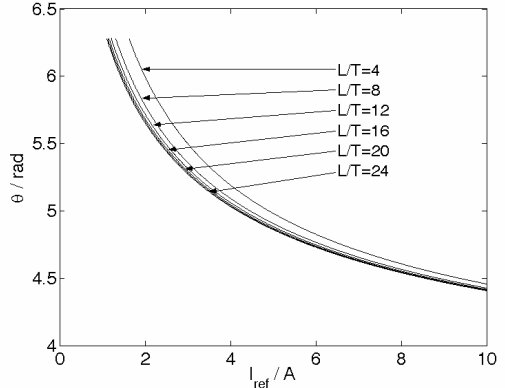

(c)

Fig. 5: The optimal initial phase $\theta$ of perturbation needed for chaos control versus $I_{\text {ref. }}$ (a) with $R=12,16,20,24,28,32 \Omega$; (b) with $E=6,8,10,12,14,16 \mathrm{~V}$; (c) with $\xi=4,8,12,16,20,24$

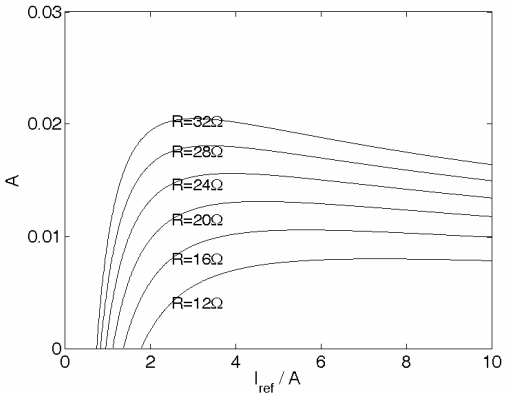

(a)

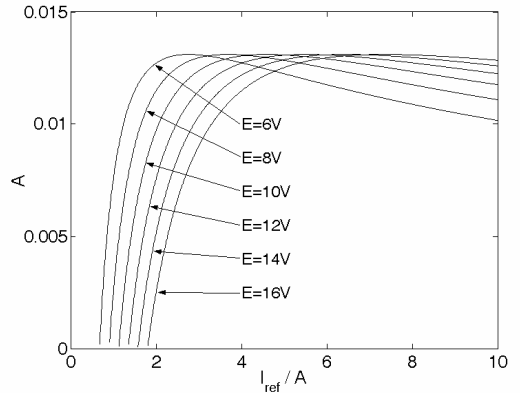

(b)

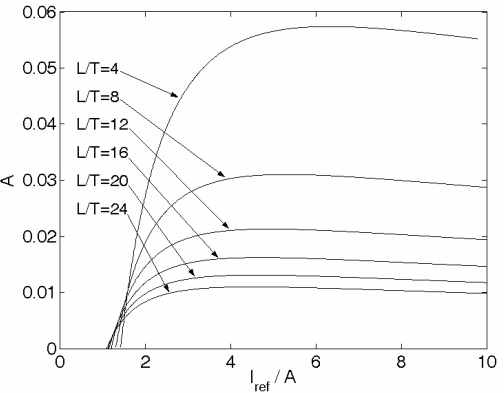

(c)

Fig. 6: The perturbation amplitude $A$ needed for chaos control versus $I_{\text {ref }}$ when optimal initial phase adopted. (a) with $R=12,16$, 20, 24, 28, $32 \Omega$; (b) with $E=6,8,10,12,14,16 \mathrm{~V}$; (c) with $\xi=4,8,12,16,20,24$

Hence, by putting $J=-1$, the critical duty cycle, at which the first period-doubling occurs, is obtained, i.e.,

$$
D_{c}=\frac{M_{c}+0.5}{M_{c}+1}
$$

Using (3) and the above expression for $D_{\mathrm{c}}$, we get the critical value of $I_{\text {ref }}$ for the perturbed system as:

$$
I_{\text {ref }}<\frac{E}{R}\left[\frac{R}{2 \xi} \frac{M_{c}+0.5}{M_{c}+1}+4\left(M_{c}+0.5\right)\left(M_{c}+1\right)\right]=I_{\text {ref }, \mathrm{c}}
$$

When the original current reference $I_{\text {ref }}$ is known, we can derive the amplitude $A$ and initial phase $\theta$ of the effective perturbation required. First, we can find the critical value of $M_{\mathrm{c}}$ by solving (16) for every known $I_{\text {ref }}$ Second, because the expression of $M_{\mathrm{c}}$ does not include the duty cycle $D$, using (13), we can easily derive the critical perturbation amplitude $A$ corresponding to stable operation for every known $I_{\text {ref. }}$. Then, the initial phase $\theta$ for the effective perturbation can also be calculated from (6) with $D$ being obtained from (15) in advance. All parameters of the circuit will affect the effective values of $A$ and $\theta$. We have summarized in Figs. 5 and 6 the dependence of the choice of the effective perturbation and optimal initial phase upon load $R$, input voltage $E$, and $\xi$ $=L / T$.

\section{CONCLUSION}

The method of resonant parametric perturbation is a nonfeedback chaos control method that is suitable to control chaos in non-autonomous systems. In this paper we have developed an optimal strategy of resonant parametric perturbation to stabilize an unstable orbit in a chaotically operating switching converter, with minimal control power.

\section{REFERENCES}

[1] C. K. Tse and M. di Bernardo, "Complex behavior in switching power converters," Proc. IEEE, 90(5): 768-781, 2002.

[2] Y. Zhou, C. K. Tse, S. S. Qiu and F. C. M. Lau, "Applying resonant parametric perturbation to control chaos in the buck de/dc converter with phase shift and frequency mismatch considerations," Int. J. Bifur. Chaos, 13(11):3459-3471, 2003.

[3] J. Wu, M. Liu and P. Yang, "Study of bifurcation and chaos in the current-mode controlled buck-boost dc-dc converter," Control Theory and Applications, 19(3):387-394, 2002.

[4] R. Lima and M. Pettini, "Suppression of chaos by resonant parametric perturbations," Phys. Rev. A, 41:726-733, 1990.

[5] C. K. Tse and Y. M. Lai, "Control of bifurcation in currentprogrammed DC/DC converters: a reexamination of slope compensation," IEEE ISCAS, Geneva Switzerland, pp. I-671-674, June 2000.

[6] Z. Qu, G. Hu, G. Yang and G. Qin, "Phase effect in taming nonautonomous chaos by weak harmonic perturbations," Phys. Rev. Lett., 74(10):1736-1739, 1995. 